\title{
Thrombocytopenia and Coronary Artery Disease, the Existing Dilemmas
}

\author{
Ilia Davarashvili, Tal Hasin, Elad Asher \\ Jesselson Integrated Heart Center, Shaare Zedek Medical Center, Jerusalem, Israel \\ Hebrew University Medical School
}

\section{ABSTRACT}

Background: Platelets play a pivotal role in the pathogenesis of acute coronary syndrome (ACS) and acute or chronic complications following percutaneous coronary intervention (PCI) as well. Platelet inhibition is a cornerstone treatment in the management of these patients. Thrombocytopenia in patients with ACS is uncommon. Idiopathic thrombocytopenic purpura (ITP) is a rare phenomenon; nevertheless, some case series presenting concomitant ACS and ITP have been described in the literature. The safety of antiplatelet therapy and PCI in patients who have ACS and thrombocytopenia is limited. Case summary: We present a case of a 60-year-old patient with ITP who was admitted with unstable angina pectoris. On admission, the platelet count was $23 \times 10^{9} / \mathrm{L}$. Coronary CT angiography revealed severe stenosis in the mid portion of RCA. After one-week treatment with high-dose Prednisolone, the platelet count recovered, and coronary catheterization was performed. Successful PCI to the RCA with drug-eluting stent was performed. The patient was discharged on dual antiplatelet therapy. Conclusion: The case suggests that PCI is a suitable treatment for ITP patients with ACS. Hemostasis is the major concern in managing these patients. The treatment strategy may be based on platelet function rather than platelet count alone. Further analysis of antiplatelet therapies as mono or dual therapy are needed.

Keywords: acute coronary syndrome, thrombocytopenia, idiopathic thrombocytopenic purpura

\section{ARTICLE HISTORY}

Received: June 13, 2019

Accepted: July 13, 2019

\section{CORRESPONDENCE}

\section{Ilia Davarashvili}

Jesselson Integrated Heart Center Shaare Zedek Medical Center P.O.B 3235, Jerusalem, Israel Tel: +972 26555955

E-mail: idavarashvili@yahoo.com

\section{BACKGROUND}

Platelets play a pivotal role in the pathogenesis of acute coronary syndrome (ACS) and acute or chronic complications following percutaneous coronary intervention (PCI) as well. Platelet inhibition is a cornerstone treatment in the management of these patients. Thrombocytopenia in patients with ACS is uncommon. Idiopathic thrombocytopenic purpura (ITP) is a rare phenomenon; nevertheless, some case series presenting concomitant ACS and ITP have been described in the literature. The safety of antiplatelet therapy and PCI in patients who have ACS and thrombocytopenia is limited.

\section{CASE PRESENTATION}

A 60-year-old Caucasian male with a history of hypertension, hypercholesterolemia, diabetes mellitus, and family history of ischemic heart disease was admitted to our hospital with an increasing chest pain and dyspnea on exertion and sometimes even at rest. Additionally, he was also known to suffer from chronic asymptomatic ITP during the past twenty years, and stable platelet count was around $30 \times 10^{9} / \mathrm{L}$ without any medical treatment. Four years prior to the index admission, he underwent computed tomography (CT) angiography due to chest pain, which revealed non-significant narrowing in the 


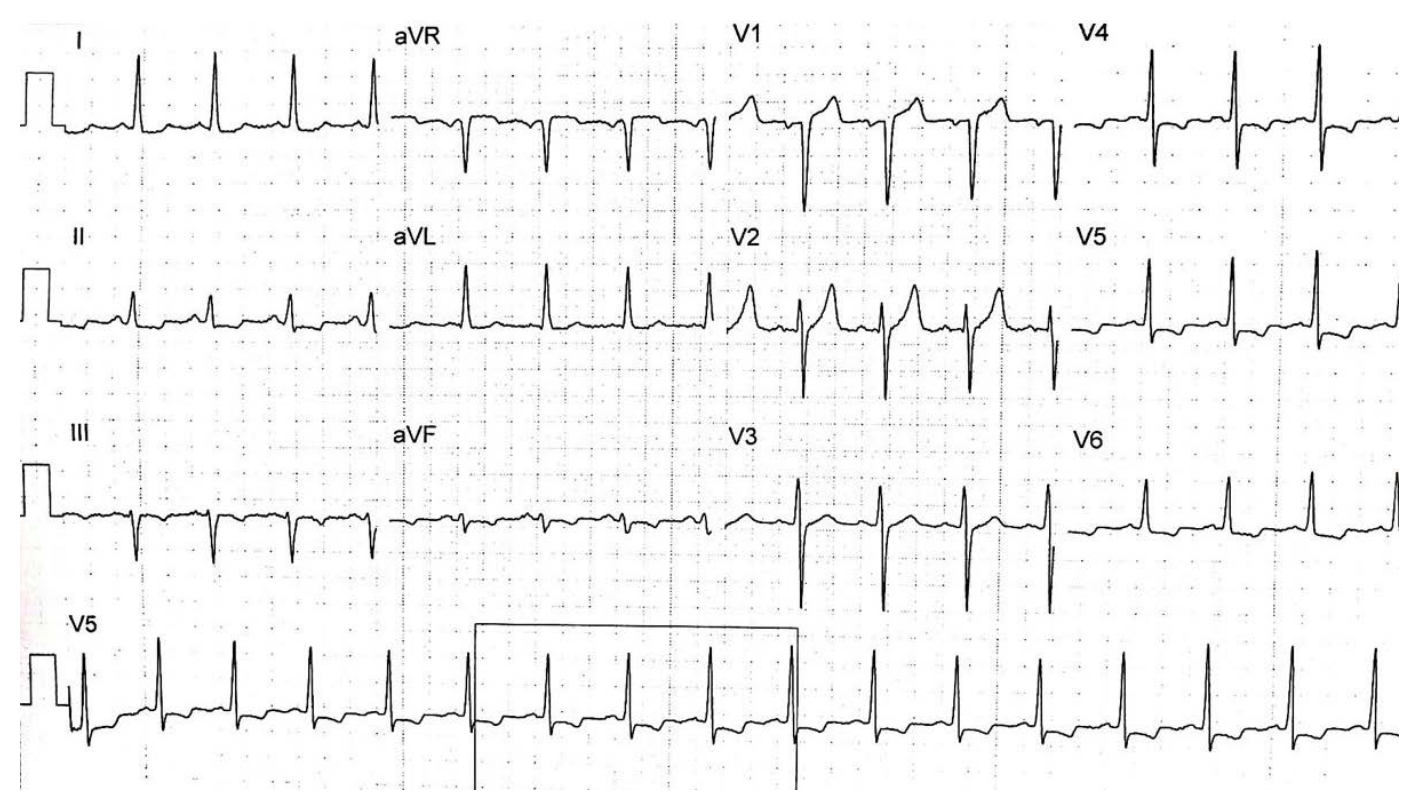

FIGURE 1. A 12-lead ECG showing normal sinus rhythm with slight ST depression and $T$ wave inversion in I, II, III, AVL, V4-6

left anterior descending (LAD) and right coronary arteries. On admission, his blood pressure was 153/88 mmHg, his heart rate was regular, $98 \mathrm{bpm}$, and physical findings were unremarkable. A 12-lead electrocardiogram (ECG) showed normal sinus rhythm, $100 \mathrm{bpm}$, with slight ST depression and $\mathrm{T}$ wave inversion in leads I, II, III, AVL, V4-6 (Figure 1). Complete blood count revealed a platelet count of $23 \times 10^{9} / \mathrm{L}$ with mean platelet volume of $13.6 \mathrm{fl}$ (normal range: 6.5-11.0), whereas cardiac enzymes and blood coagulation tests were within normal range. Chest $\mathrm{X}$-ray was unremarkable.

A diagnosis of unstable angina pectoris (UAP) was established, and the patient was treated with bisoprolol and atorvastatin. Subcutaneous enoxaparin $40 \mathrm{mg} / \mathrm{d}$ was administered following hematologic consultation. Coronary CT angiography was performed, revealing a severe ste-
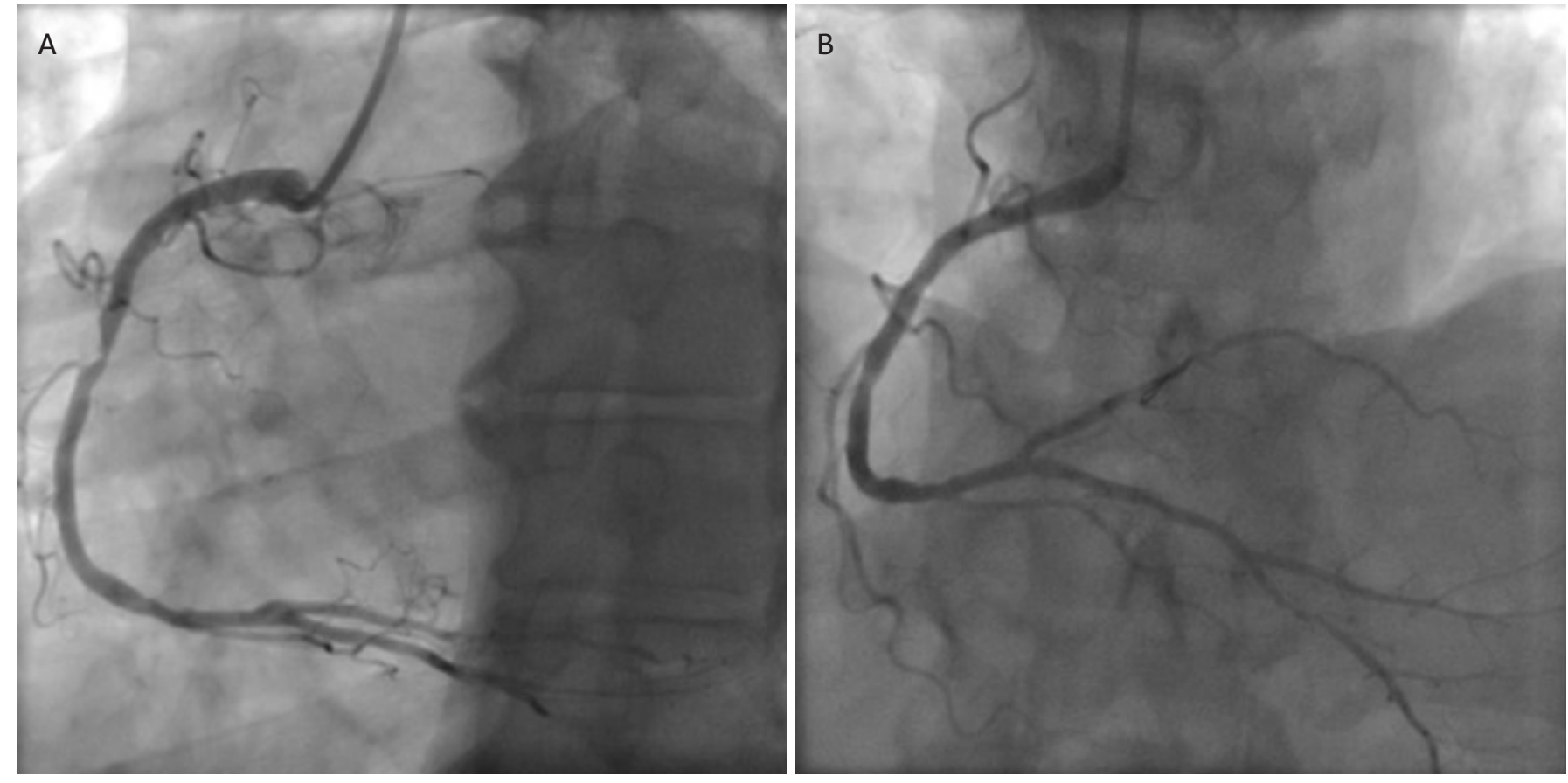

FIGURE 2. Coronary angiography of the RCA before $\mathrm{PCI}(\mathbf{A})$, after $\mathrm{PCI}(\mathbf{B})$ 
nosis in the mid portion of RCA and a calcified LAD with a moderate stenosis. Transthoracic echocardiography demonstrated moderately reduced left ventricular function. During the first days of hospitalization, the patient was treated with prednisolone $80 \mathrm{mg}$ daily; five days later, the platelet count rose to $61 \times 10^{9} / \mathrm{L}$, and aspirin monotherapy was initiated. On day 7 , the platelet count rose to $106 \times 10^{9} / \mathrm{L}$, and coronary angiogram was performed via the radial artery, revealing double-vessel disease with $60 \%$ stenosis of the mid LAD and $80 \%$ of the mid RCA. Successful PCI to the RCA with drug-eluting stent was performed (Figure 2). The patient signed a written informed consent, in which he approved the publication of his medical information, and the study procedures were performed according to the ethical principles stated in the Declaration of Helsinki. Publication of this case was also approved by the ethics committee of the hospital.

\section{DISCUSSION}

Dual antiplatelet therapy (DAPT) consisting of aspirin and a P2Y12 receptor antagonist is a fundamental component of ACS management. ${ }^{1,2}$ The management of antiplatelet therapy in ACS patients with thrombocytopenia poses a particular challenge for physicians, as they are at higher risk of both bleeding and, paradoxically, ischemic events. ${ }^{3-5}$

The cause of coronary thrombosis in patients with thrombocytopenia is debatable and multifactorial. Independently of their thrombocytopenia, patients may be predisposed to coronary thrombosis because their platelets are larger and more adhesive to the vascular surface. ${ }^{6-8}$ Evidence supports the concept that large platelets have a higher thrombotic potential. ${ }^{9}$ Because platelet production is naturally regulated to maintain platelet mass (the product of platelet count and mean platelet volume), it can be inferred that the larger platelets not only provide a hemostatic plug and prevent bleeding via high activity, but also that they may be linked to coronary thrombosis in patients with thrombocytopenia. There is substantial evidence that microparticles derived from platelets play a role in the activation of the coagulation cascade. 9 Levels of platelet microparticles are significantly higher in patients who have ITP than in control groups as well as in ACS patients. ${ }^{10,11}$ There are few reports, mainly on critically ill hemato-oncological patients, which describe the use of antiplatelet agents with no bleeding sequelae thrombocytopenia. ${ }^{12,13}$

Antiplatelet therapy in the presence of thrombocytopenia is debatable, and there are no widely accepted protocols. ${ }^{14}$ The current recommendation is to avoid giving antiplatelet agents to patients with ACS who have severe thrombocytopenia (i.e. platelet count $<50 \times 10^{9} / \mathrm{L}$ ). ${ }^{15}$ The presence of thrombocytopenia in ACS patients predicts significantly worse outcomes. ${ }^{16,17}$ The association of thrombocytopenia and increased ischemic events remains poorly understood. It may reflect the presence of a greater degree of comorbidities that are unmeasured. ${ }^{18,19}$ Which DAPT is preferable in these patients is still unknown. Clopidogrel offers a lower bleeding risk than ticagrelor or prasugrel, and, accordingly, would be the agent of choice in patients receiving DAPT. ${ }^{3}$ Management should begin with the identification and correction of any reversible causes of thrombocytopenia. ${ }^{18}$ It is advised to avoid prasugrel and ticagrelor due to their association with higher bleeding rates, although ticagrelor monotherapy, with its twice daily dosing and reversible binding, would be of great interest. ${ }^{15}$ Thrombocytopenia may be associated with more concurrent diseases and mechanisms of pro-thrombosis, including ACS. Approaches to the treatment of ACS in thrombocytopenic patients might be better directed toward the evaluation of platelet function than toward platelet count. ${ }^{20}$ No doubt, the management of immune thrombocytopenic purpura and acute coronary syndrome is a double-edged sword. ${ }^{13}$ However, thrombocytopenia is somehow reversible with the mainstay of the treatment: prednisolone, azathioprine, IVIG, and romiplostim. ${ }^{13,21}$

Our patient was treated by high-dose prednisone, and successful PCI to RCA was performed. He was discharged with high-dose steroids and DAPT for 6 months. No bleeding occurred during follow-up, and the platelet count level was stabilized around $70 \times 10^{9} / \mathrm{L}$. The treatment of the ITP was switched to azathioprine with a good response.

\section{CONCLUSIONS}

Patients with ITP and concomitant ACS are rare; nevertheless, definite treatment guidelines for these patients have not been established. We have performed coronary CTA first in order to establish the definite diagnosis and avoid an unnecessary invasive procedure, especially when the urgent procedure cannot be performed and while correcting the hematologic parameters. The case we have reported suggests that PCI is a suitable treatment for ITP patients with ACS. Hemostasis is the major concern in managing these patients. The risk and benefit of long-term antiplatelet agent use should be considered in each patient and should be individualized. The treatment strategy may be based on platelet function rather than on platelet count alone. Further analysis of antiplatelet therapies as mono or dual therapy are needed. 


\section{CONFLICT OF INTEREST}

Nothing to declare.

\section{REFERENCES}

1. Levine GN, Bates ER, Bittl JA, et al. 2016 ACC/AHA guideline focused update on duration of dual antiplatelet therapy in patients with coronary artery disease: A report of the American College of Cardiology/American Heart Association task force on clinical practice guidelines: An update of the 2011 ACCF/AHA/SCAI guideline for percutaneous coronary intervention, 2011 ACCF/AHA guideline for coronary artery bypass graft surgery, 2012 ACC/AHA/ACP/AATS/PCNA/SCAI/ STS guideline for the diagnosis and management of patients with stable ischemic heart disease, 2013 ACCF/AHA guideline for the management of ST-elevation myocardial infarction, 2014 AHA/ACC guideline for the management of patients with non-ST-elevation acute coronary syndromes, and 2014 ACC/ AHA guideline on perioperative cardiovascular evaluation and management of patients undergoing noncardiac surgery. Circulation. 2016;134:e123-e155. doi: 10.1161/ CIR.0000000000000404.

2. Ibanez B, James S, Agewall S, et al. ESC guidelines for the management of acute myocardial infarction in patients presenting with ST-segment elevation, the task force on the management of ST-segment elevation acute myocardial infarction of the European Society of Cardiology (ESC). Eur Heart J. 2018;39:119-177. doi: 10.1093/eurheartj/ehx393.

3. Bhatt DL, Hulot JS, Moliterno DJ, Harrington RA. Antiplatelet and anticoagulation therapy for acute coronary syndromes. Circ Res. 2014;114:1929-1943. doi: 10.1161/CIRCRESAHA.114.302737.

4. Udell JA, Bonaca MP, Collet JP, et al. Long-term dual antiplatelet therapy for secondary prevention of cardiovascular events in the subgroup of patients with previous myocardial infarction: A collaborative meta-analysis of randomized trials. Eur Heart J. 2016;37:390-399. doi: 10.1093/eurheartj/ehv443.

5. McClure MW, Berkowitz SD, Sparapani R, et al. Clinical significance of thrombocytopenia during a non-ST-elevation acute coronary syndrome. The platelet glycoprotein IIb/IIIa in unstable angina: Receptor suppression using integrilin therapy (PURSUIT) trial experience. Circulation. 1999;99:2892-2900. doi: 10.1161/01.cir.99.22.2892.

6. Cameron HA, Phillips R, Ibbotson RM, Carson PH. Platelet size in myocardial infarction. Br Med J (Clin Res Ed). 1983;287:449451. doi: 10.1136/bmj.287.6390.449.

7. O'Malley T, Langhorne P, Elton RA, Stewart C. Platelet size in stroke patients. Stroke. 1995;26:995-999.

8. Asher E, Fefer P, Shechter M, et al. Increased mean platelet volume is associated with non-responsiveness to clopidogrel. Thromb Haemost. 2014;112:137-141. doi: 10.1160/TH13-100845.
9. VanWijk Michiel, VanBavel Ed, Nieuwland R. Microparticles in cardiovascular diseases. Cardiovasc Res. 2003;59:277-287. doi: 10.1186/1471-2121-14-23.

10. Jy W, Horstman LL, Arce M, Ahn YS. Clinical significance of platelet microparticles in autoimmune thrombocytopenias. J Lab Clin Med. 1992;119:334-345.

11. Ozner MD, Ahn YS, Horstman LL, Jy W, Kolodny L, Myerberg RJ. Chronic platelet activation and acute coronary syndromes in 13 middle-aged patients. Clin Appl Thromb Hemost. 1997;3:46-53.

12. Jachmann-Jahn U, Cornely OA, Laufs U, et al. Acute anterior myocardial infarction as first manifestation of acute myeloid leukemia. Ann Hematol. 2001;80:677-681.

13. Shah AH, Anderson RA, Khan AR, Kinnaird TD. Management of immune thrombocytic purpura and acute coronary syndrome: A double-edged sword! Hellenic J Cardiol. 2016;pii:S1109-9666(16)30151-8. doi: 10.1016/j. hjc.2014.10.001.

14. Kohl BA. Con: Should aspirin be continued after cardiac surgery in the setting of thrombocytopenia? J Cardiothorac Vasc Anesth. 2006;20:114-116. doi: 10.1053/j.jvca.2005.10.007.

15. McCarthy CP, Steg G, Bhatt DL. The management of antiplatelet therapy in acute coronary syndrome patients with thrombocytopenia: a clinical conundrum. Eur Heart J. 2017;38:3488-3492. doi: 10.1093/eurheartj/ehx531.

16. Wang TY, Ou FS, Roe MT, et al. Incidence and prognostic significance of thrombocytopenia developed during acute coronary syndrome in contemporary clinical practice. Circulation. 2009;119:2454-2462. doi: 10.1161/ CIRCULATIONAHA.108.827162.

17. Yadav M, Généreux P, Giustino G, et al. Effect of baseline thrombocytopenia on ischemic outcomes in patients with acute coronary syndromes who undergo percutaneous coronary intervention. Can J Cardiol. 2016;32:226-233. doi: 10.1016/j.cjca.2015.05.020.

18. McClure MW, Berkowitz SD, Sparapani R, et al. Clinical significance of thrombocytopenia during a non-ST-elevation acute coronary syndrome. The platelet glycoprotein IIb/IIIa in unstable angina: Receptor suppression using integrilin therapy (PURSUIT) trial experience. Circulation. 1999;99:2892-2900. doi: 10.1161/01.cir.99.22.2892.

19. Warkentin TE, Crowther MA. Adverse prognostic significance of thrombocytopenia in acute coronary syndrome. Can anything be done about it? Circulation. 2009;119:2420-2422. doi: 10.1161/CIRCULATIONAHA.109.858316.

20. Yusuf SW, Iliescu C, Bathina JD, Daher IN, Durand JB. Antiplatelet Therapy and Percutaneous Coronary Intervention in Patients with Acute Coronary Syndrome and Thrombocytopenia. Tex Heart Inst J. 2010;37:336-340.

21. Schwartz RS. Immune thrombocytopenic purpura - from agony to agonist. N Engl J Med. 2007;357:2299-2301. doi: 10.1056/NEJMe0707126. 